\title{
Evaluation of LMP1 of Epstein-Barr virus as a therapeutic target by its inhibition
}

\author{
Adele Hannigan ${ }^{1,2}$ and Joanna B Wilson*1
}

\begin{abstract}
Background: The latent membrane protein-1 (LMP1) encoded by Epstein-Barr virus (EBV) is an oncoprotein which acts by constitutive activation of various signalling pathways, including NF-KB. In so doing it leads to deregulated cell growth intrinsic to the cancer cell as well as having extrinsic affects upon the tumour microenvironment. These properties and that it is a foreign antigen, lead to the proposition that LMP1 may be a good therapeutic target in the treatment of EBV associated disease. LMP1 is expressed in several EBV-associated malignancies, notably in Hodgkin's lymphoma and nasopharyngeal carcinoma (NPC). However, the viral protein is only detected in approximately 30\%$50 \%$ of NPC samples, as such its role in carcinogenesis and tumour maintenance can be questioned and thus its relevance as a therapeutic target.

Results: In order to explore if LMP1 has a continuous function in established tumours, its activity was inhibited through expression of a dominant negative LMP1 mutant in tumour cell lines derived from transgenic mice. LMP1 is the tumour predisposing oncogene in two different series of transgenic mice which separately give rise to either B-cell lymphomas or carcinomas. Inhibition of LMP1 activity in the carcinoma cell lines lead to a reduction in clonagenicity and clone viability in all of the cell lines tested, even those with low or below detection levels of LMP1. Inhibition of LMP1 activity in the transgenic B-cell lines was incompatible with growth and survival of the cells and no clones expressing the dominant negative LMP1 mutant could be established.

Conclusions: LMP1 continues to provide a tumour cell growth function in cell lines established from LMP1 transgenic mouse tumours, of both B-cell and epithelial cell origin. LMP1 can perform this function, even when expressed at such low levels as to be undetectable, whereby evidence of its expression can only be inferred by its inhibition being detrimental to the growth of the cell. This raises the possibility that LMP1 still performs a pro-oncogenic function in the $50 \%$ to $70 \%$ of NPC tumours wherein LMP1 protein expression cannot be detected. This reinforces the basis for pursuing LMP1 as a therapeutic target in EBV associated LMP1-expressing malignancies.
\end{abstract}

\section{Background}

Epstein-Barr Virus (EBV) is a human herpes virus which is associated with a number of malignant diseases reflecting the viral tropism primarily to B-cells but also to epithelial cells and rarely other cell types. The EBVassociated B-cell cancers include endemic Burkitt's lymphoma (BL), a subset of Hodgkin's disease (HD) cases and lymphoid tumours arising in immunosuppressed patients; the epithelial cell cancers include nasopharyngeal carcinoma (NPC) and a proportion of gastric cancers. EBV shows a different but typical pattern of latent

* Correspondence: joanna.wilson@bio.gla.ac.uk

${ }^{1}$ Division of Molecular and Cellular Biology, Faculty of Biomedical and Life Sciences, University of Glasgow, Glasgow G11 6NU, UK

Full list of author information is available at the end of the article gene expression in each of these malignancies, from the most restricted pattern of viral expression in $\mathrm{BL}$, to expression of all of the viral latent genes in post-transplant lymphoproliferative disease. NPC and HD biopsies show an intermediate pattern of viral gene expression involving EBNA-1, latent membrane proteins-1 and -2A (LMP1 and LMP2A), EBERs and the BART micro RNAs [1].

LMP1 exhibits properties of a classical oncoprotein, inducing promotion of cell growth and inhibition of apoptosis in a variety of cell types in vitro [2]. In addition it has been demonstrated to contribute to both B-cell and epithelial cell tumourigenesis in vivo in transgenic mice [3-5]. LMP1 achieves its wide ranging phenotypic effects through the activation of multiple signalling cascades. It 
activates the NF-kB, JNK and JAK/STAT pathways through direct interaction with pathway intermediary proteins [6]. As a consequence of the gene expression changes induced, for example affecting EGFR and it's ligands $[7,8]$, further pathways are triggered including the ERK/MEK and p38/MAPK pathways. As such, LMP1 is considered as the primary oncogene of the virus and a likely candidate in driving the development of several of the EBV associated malignancies.

Significant progress has been made in recent years in cancer therapeutics in the design of inhibitory molecules that impact relevant signalling pathways, for example BRaf inhibition in the treatment of melanoma [9]. As a foreign antigen that constitutively activates multiple pathways, LMP1 represents a good therapeutic target in the treatment of EBV associated malignancies. Moreover, while LMP1 activates growth pathways within the cancer cell, in deregulating NF- $\mathrm{kB}$ it also impacts a seminal pathway in inflammation programmes and thus potentially, factors in the tumour microenvironment. Therefore targeting LMP1 could affect both intrinsic and extrinsic factors essential to tumour growth. LMP1 expression has been confirmed by immunohistochemical studies in EBV-associated HD. However, detection of LMP1 protein in NPC biopsies is highly variable, with only between $30 \%$ to $50 \%$ of tumours showing clear expression [10] despite the detection of LMP1 RNA in most samples. Indeed it has been shown that the BART micro RNAs of the virus, which are abundantly expressed in NPC, negatively regulate LMP1 protein expression [11]. This raises some uncertainty about the role of LMP1 in the genesis of NPC and particularly any tumour maintenance function, especially in those tumours where expression cannot be detected. This in turn poses the question of whether LMP1 is a rational therapeutic target.

Inhibition of LMP1 expression by siRNA in an EBV positive NPC derived cell line C666-1, which clearly expresses LMP1, was found to induce cell cycle arrest and enhance the sensitivity of the cells to cisplatin [12]. This observation is encouraging with respect to LMP1 as a potential therapeutic target. However it is unknown at present if this finding will be limited to those NPC tumours with high LMP1 expression. In this study we sought to evaluate the impact of LMP1 inhibition in multiple cell lines, of both epithelial and B-cell origin where LMP1 was the driving oncogene in the development of the tumour. It is notoriously difficult to derive cell lines from NPC and HD tumours and as a consequence there are few lines available. To this end we used cell lines derived from tumours from transgenic mice where LMP1 was the predisposing oncogene. These lines were also used with a view to future in vivo drug testing. In all of the LMP1 transgenic cell lines tested, inhibition of LMP1 activity inhibited the growth properties of the cells (to varying extents in the different lines) surprisingly even in those where LMP1 protein expression was not detectable. Firstly, this demonstrates that even extremely low levels of LMP1 can continue to provide a growth advantage to cancer cells and secondly, as a consequence, its inhibition could be an effective route in the treatment to eliminate the cells. However in one highly malignant carcinoma cell line, inhibition of LMP1 lead to the selection of escape mutants indicating that any treatment targeting LMP1 would be best used as part of a combined therapy regime.

\section{Results}

\section{LMP1 expression in transgenic carcinoma and lymphoma}

\section{cell lines}

In order to investigate the tumour growth promoting properties of LMP1 and whether its continued expression is required in established tumours, carcinomas and B-cell lymphomas from LMP1 expressing transgenic mice were established in culture.

Carcinomas were induced in transgene positive and negative sibling controls (NSC) in the transgenic PyLMP1 line 53 (PyLMP1.53) [3], by topical treatment with chemical carcinogens [13]. These tumours could be readily established in culture; some retained a cuboidal, squamous morphology while others developed a spindle morphology with more transformed growth characteristics (additional files 1\&2, supplementary table S1). LMP1 was difficult to extract from these epithelial cells (as with transgenic skin tissue [5]), suggesting an association with the cytoskeleton and necessitating the use of a urea extraction protocol. LMP1 expression was detected by immunoprecipitation and western blotting in several, but not all of the transgene positive carcinoma cell lines developed (figure 1A). Nevertheless, the cell lines in which expression could not be detected maintained the transgene (not shown). There was no apparent correlation between the carcinoma grade, cell line phenotype and LMP1 expression. For example, cell line 53.278a, derived from an aggressive spindle cell carcinoma and showing rapid spindle cell growth in culture (see supplementary information table 1) showed LMP1 expression as did the more cuboidal cell line 234a (with highest LMP1 expression) derived from a grade 3 carcinoma. However, with cuboidal cell line 53.226b (grade 1 carcinoma) and spindle cell line 53.191 (grade 3 carcinoma), little or no LMP1 expression could be detected.

Lymphomas arise spontaneously in aged mice of the transgenic line E $\mu \mathrm{LMP1} 139$ (additional files 1\&2, supplementary figure S1) in which LMP1 expression is directed to the lymphoid compartment [3]. Cell line 39.415 is a murine B-cell line developed from a lymphoma from transgenic line E $\mu$ LMP1.39 (additional files 1\&2, supplementary figure S2) showing readily detectable LMP1 expression (figure 1B). LMP1 expression in the 39.415 cell 
Table 1: Clonagenicity of the epithelial cell lines with dnLMP1

\begin{tabular}{|c|c|c|c|c|}
\hline \multirow[t]{2}{*}{ cell line } & \multirow{2}{*}{$\begin{array}{c}\text { transgene } \\
\text { status }\end{array}$} & \multicolumn{2}{|c|}{ colonies per $\mu \mathrm{g}$ of DNA } & \multirow{2}{*}{$\begin{array}{c}\text { colony ratio } \\
\text { pGFPdnLMP1:pGFP }\end{array}$} \\
\hline & & pGFPdnLMP1 & pGFP & \\
\hline 53.217 & - & 164 & 170.4 & $1: 1.0$ \\
\hline $53.234 a$ & + & 8.8 & 41.6 & $1: 4.7$ \\
\hline 53.204 & + & 0 & 4.2 & - \\
\hline 53.191 & + & 14 & 42 & $1: 3.0$ \\
\hline $53.226 b$ & + & 1.6 & 6 & $1: 3.8$ \\
\hline $53.278 \mathrm{a}$ & + & 46 & 112 & $1: 2.4$ \\
\hline $53.278 b$ & + & 1.2 & 13.2 & $1: 11.0$ \\
\hline
\end{tabular}

line is approximately 30-fold lower than the human $\mathrm{BL}$ cell line Raji (which harbours 50 to 60 copies of the EBV genome per cell). Cell line 3959.48 was established from a $\mathrm{B}$-cell lymphoma arising in a bi-transgenic mouse harbouring E $\mu \mathrm{LMP} 1$ and $\mathrm{E} \mu \mathrm{EBNA}-1$ transgenes. It expresses readily detectable EBNA1 [14] and low levels of LMP1, with the latter at least 300-fold lower (on a per cell basis) than cell line 39.415 (figure 1B). Cell line 39.415 tends to grow in large clumps in culture, while 3959.48 grows as a single cell suspension or in small clumps, possibly reflecting LMP1 induced homotypic adhesion [15] and their relative levels of LMP1.

\section{Inhibition of LMP1 in the transgenic carcinoma cell lines}

In order to inhibit LMP1 activity a dominant negative mutant of LMP1 which is defective in the LMP1 induced signalling pathways, termed LMP1AAAG, fused to GFP [16] denoted here as GFPdnLMP1 (or dnL as designation for transfected cells and clones) was introduced into the transgenic carcinoma cell lines. Using the parental GFP expression vector as control, six PyLMP1 transgenic carcinoma cell lines were transfected and one transgene negative control (figure 2, table 1). Following 2 weeks of plasmid selection, in all PyLMP1 cell lines the number of clones derived from pGFPdnLMP1 transfection was less than that from pGFP transfection, ranging from a 2.4 fold difference for (line 53.278a) to an 11 fold difference (line $53.278 \mathrm{~b}$ ) and in one cell line (53.204) no GFPdnLMP1 clones emerged. Furthermore, the pGFPdnLMP1 transfected clones tended to be smaller and less dense than the
pGFP transfectants (figure 2). In contrast, clones of equivalent size and density were obtained in equal numbers for the two plasmids in the transgene-negative carcinoma cell line 53.217 (figure 2, table 1). This demonstrates that the pGFPdnLMP1 and pGFP plasmids were not toxic and of equal impact (if any) in an LMP1 negative carcinoma cell line. However, the data suggest that in all of the PyLMP1 transgenic cell lines, even those where LMP1 expression was low or undetectable (cell lines $53.278 \mathrm{~b}, 53.226 \mathrm{~b}, 53.191)$, dnLMP1 is inhibitory to clonagenicity. Clones derived in this manner were either cultured as a pool or individually isolated for further analysis from the transgene negative cell line 53.217 and two PyLMP1 positive cell lines 53.234a and 53.278a.

Only one of six GFPdnLMP1 53.234a clones isolated could be established (referred to as 53.234dnL-1) while all six $53.217 \mathrm{dnL}$ clones were expanded. 10/12 clones of $53.278 \mathrm{adnL}$ were also established. This again reflects the inhibitory effect of dnLMP1 upon the clonagenicity of cell line 53.234a and to a lesser extent with cell line 53.278a. GFPdnLMP1 expression was confirmed in the single $53.234 \mathrm{dnL}-1$ clone and in $3 / 3$ tested $53.217 \mathrm{dnL}$ clones (figure 3A). For 53.278adnL clones, 5/10 showed clear GFPdnLMP1 expression (figure $3 \mathrm{~B}$ and $3 \mathrm{C}$ and additional files $1 \& 2$, supplementary figure S3). GFP expression was confirmed in the majority of control pGFP transfected clones tested (figure 3).

The single 53.234dnL-1 clone established must have selectively overcome the inhibitory effect of dnLMP1 to some degree. In order to explore this further, clone 


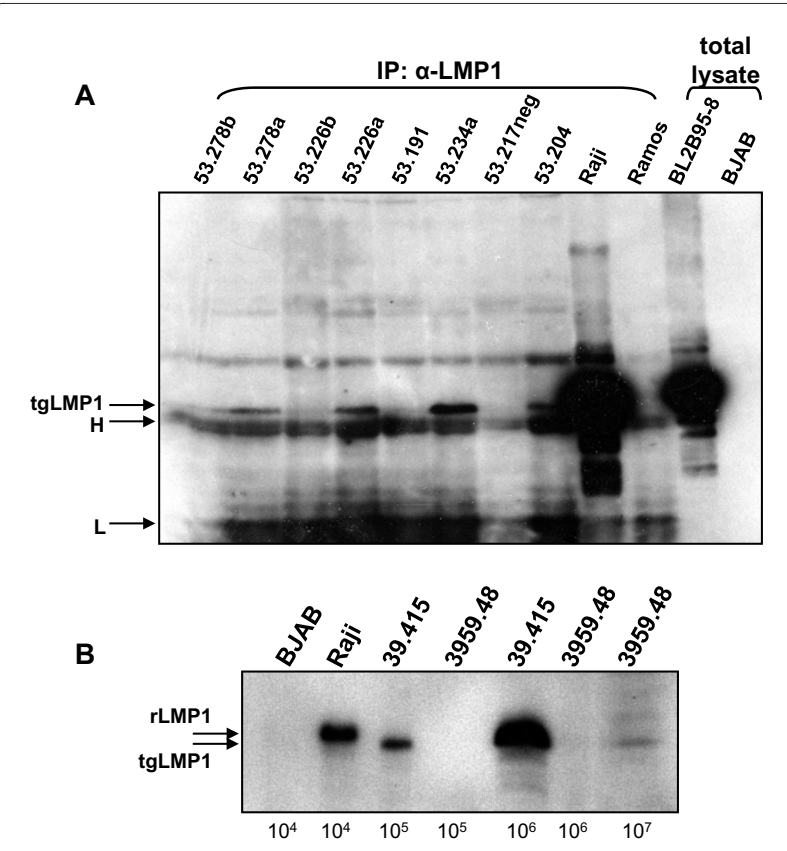

Figure 1 LMP1 expression in transgenic mouse derived cell lines. (A) LMP1 was immunoprecipitated from $300 \mu \mathrm{g}$ of urea buffer-extracted proteins from seven PyLMP1 transgene-positive line 53 carcinoma cell lines and one transgene-negative cell line (as denoted 53.217neg) and $100 \mu \mathrm{g}$ of protein from control cell lines: Raji (EBV +ve, containing 50-60 copies of EBV) and Ramos (EBV -ve), using the S12 antiserum. Immunoprecipitated proteins were separated by 10\%SDS-PAGE and blotted alongside two total lysate (boiling mix extracted) controls, BL2B95-8 (EBV +ve) and BJAB (EBV-ve) $\left(1 \times 10^{5}\right.$ cells each). The blot was probed with anti-LMP1 antibody $1 \mathrm{G} 6$ followed by goat a-rat lgG HRP. The bands corresponding to LMP1 and the immunoglobulin heavy $(H)$ and light $(\mathrm{L})$ chains are indicated on the left. (B) Protein was extracted from B-cell lines 39.415 and 3959.48 with controls Raji and BJAB (the number of cells indicated below each track) using boiling mix and samples western blotted for LMP1 using IG6. The transgenic LMP1 (tgLMP1) is slightly smaller than Raji LMP1 (rLMP1) [3].

$53.234 \mathrm{dnL}-1$ was compared to clone $53.217 \mathrm{dnL}-3$ (with highest expression of the 53.217 clones) for cell growth, against the parental cell lines and clones expressing only GFP. With the transgene negative cell line 53.217, clones expressing GFP or GFPdnLMP1 showed identical growth curves compared to the parental cell line (figure 4). However, the PyLMP1 positive clone 53.234dnL-1 showed significantly slower growth compared to both the parental cell line and GFP transfectants (figure 4). These data suggest that despite clone 53.234dnL-1 having been established under the selective pressure of dnLMP1 expression, i.e. inhibition of LMP1, the growth is nevertheless impaired compared to the parental cell line. Thus any genetic or epigenetic changes that have occurred in this cell clone to allow it to become established have not fully compensated for the blockade of LMP1 activity in cell growth.

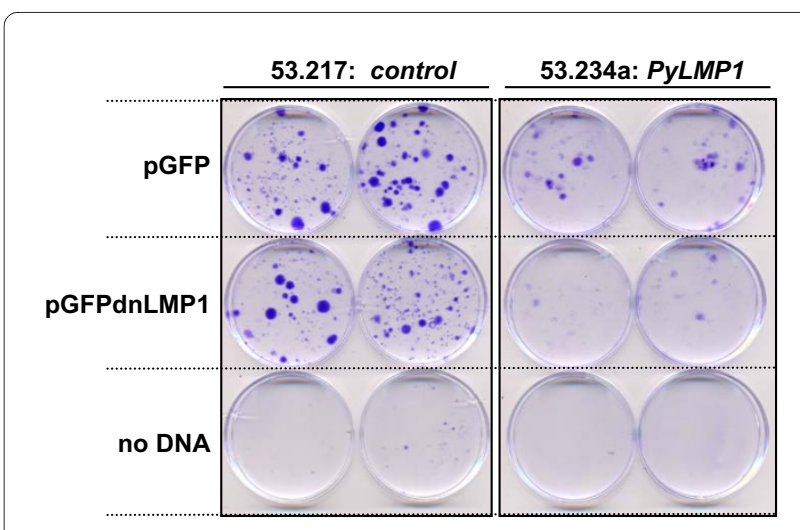

Figure 2 GFPdnLMP1 inhibits the clonogenicity of PyLMP1 transgenic carcinoma cell lines. Cell lines 53.217 (transgene negative control) and 53.234a (PyLMP1 transgenic) were transfected with $5 \mu \mathrm{g}$ of either pGFP or GFPdnLMP1 or mock transfected (no DNA) in duplicate. After 24 hrs cells were passaged 1 to 8 and cultured for two weeks under $\mathrm{G} 418$ selection before fixing and staining with crystal violet. Colony numbers for these and further cell lines treated in the same way are given in Table 1.

We then examined the aggressive spindle cell line 53.278a which had shown least dependency upon LMP1 in the clonagenicity assay (table 1). Growth of three of the clones showing highest GFPdnLMP1 expression (53.278adnL-4, -6 and -8) were compared to the parental cell line and the highest GFP expressing control clone. The GFP clone 53.278aGFP-5 showed an identical growth rate to the parental cell line, while all three dnLMP1 clones revealed significantly accelerated growth rates (figure 4). These data demonstrate that enforced dnLMP1 expression in this cell line has selected for more rapidly growing clones presumably independent of LMP1 activity. The clone with highest GFPdnLMP1 expression, clone 53.278dnL-8 was assessed for tumourigenicity compared to the parental cell line, using syngeneic recipient mice. The clone retained the tumourigenic phenotype and in 3/4 subsequently derived tumours GFPdnLMP1 expression was maintained (additional files 1\&2, supplementary figure S4).

\section{Inhibition of LMP1 in the transgenic B-cell lines}

Inhibition of LMP1 activity in the tumour derived B-cell lymphoma cells lines 39.415 and 3959.48 was similarly assessed by transfection of the GFPdnLMP1 or GFP expression vectors. The antibiotic selection process was complete by 3 weeks post transfection at which point the cell lines were assayed for GFPdnLMP1 and GFP expression. Cells were harvested at weekly intervals for four weeks maintaining drug selection. With 39.415 cells, GFP expression could be detected in the control pGFP transfectants consistently for the four week period (figure 5A). However while clear GFPdnLMP1 expression was 


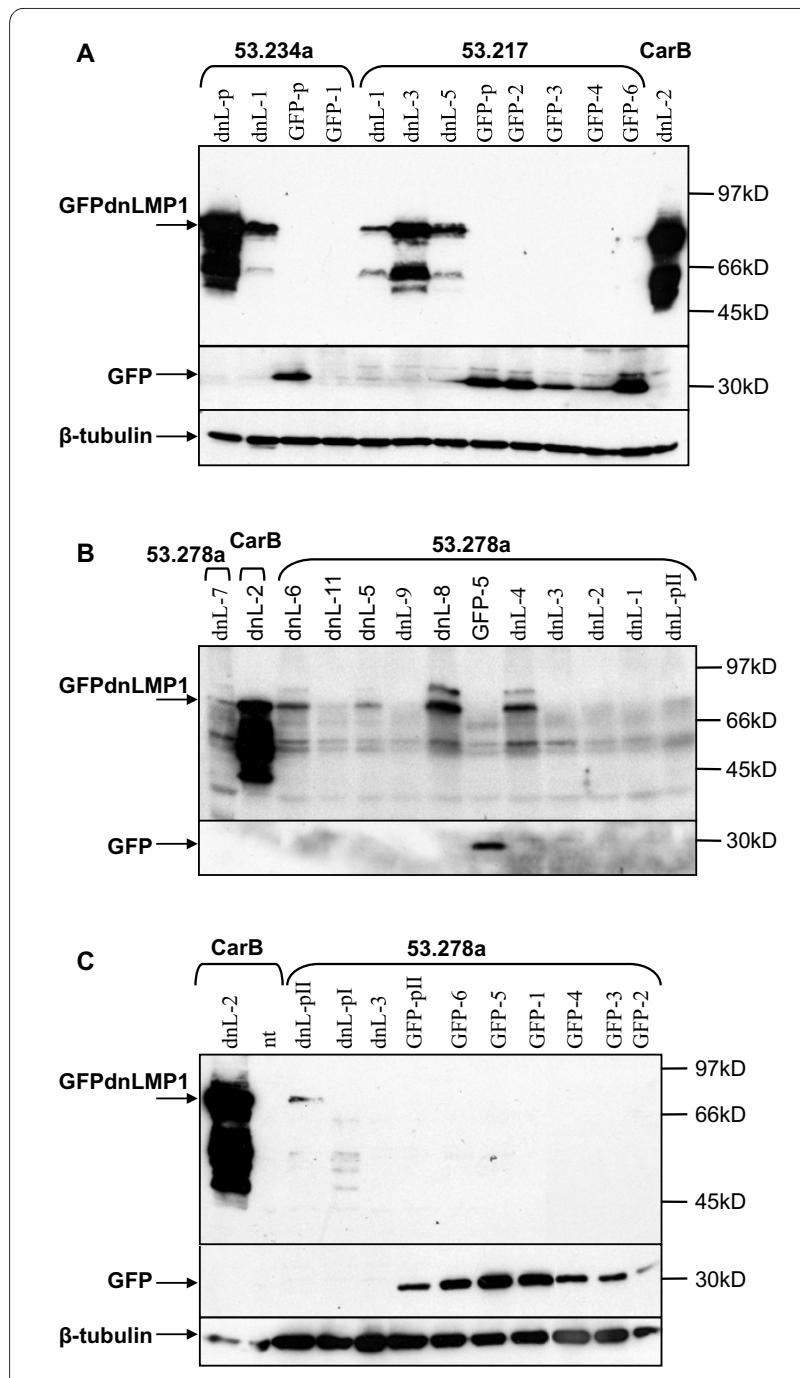

Figure 3 Expression of GFP and GFPdnLMP1 in transfected carcinoma cells. Transgene negative control cell lines 53.217 and CarbB, and transgenic PyLMP1 cell lines 53.234a and 53.278a, transfected with pGFP or pGFPdnLMP1 were sub-cloned to give clones denoted GFP and $\mathrm{dnL}$ (respectively). Protein samples from each cell line sub-clone (indicated by -number) or pool of clones (-p) were examined by western blotting, sequentially using anti-LMP1 (top of each panel), a-GFP (middle) and a-beta-tubulin (bottom), as indicated. (A) $100 \mathrm{\mu g}$ of protein extract from sub-clones or pools of clones from the cell lines 53.217, CarB and 53.234a. (B) and (C) $50 \mu \mathrm{g}$ protein extract from subclones or pools of clones ( $\mathrm{pl}$ and PII) from the cell lines 53.278a and control CarB. Non-transfected parental cell line (nt)

detected at 3 and 4 weeks post transfection, it disappeared from the transfected culture by 5 weeks post transfection. Similarly, clear green fluorescence could be seen in the pGFP transfectants ( 3 weeks post transfection) but only weak fluorescence in the pGFPdnLMP1 39.415 transfectants (figure 5B). In contrast, green fluorescence in both pGFP and pGFPdnLMP1 transfectants of the control EBV negative cell line AK31 was clearly visible (additional files 1\&2, supplementary figure S5) and

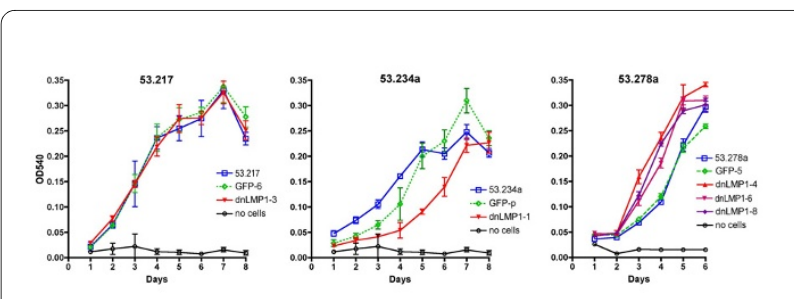

Figure 4 Growth curves of GFP and GFPdnLMP1 transfected carcinoma clones. Clones of the transfected cell lines 53.217 (transgene negative; left graph), 53.234a (PyLMP1 transgene; middle graph) and 53.278a (Py LMP1 transgene; right graph) that showed the highest expression of GFP or GFPdnLMP1 were analysed for growth by neutral red assay and compared to the parental cell line in each case. Means of the four replicates (with SD) are plotted. Clone 53.234adnL-1 shows significantly different values from the parental cell line 53.234a (day 3 : $p<0.0001$, day 4: $p<0.0001$, day $5: p<0.0001$, day $6: p=0.0009$ ). Similarly 53.278a clone values differ significantly from the parental 53.278a cell line (eg. for clone 53.278adnL-8: day 3: $p=0.0003$, day 4: $p<0.0001$, $d 5: p=0.002$ )

could consistently be detected by western to at least 12 weeks after transfection (figure 5C). With the 3959.48 cell line, similarly consistent GFP expression was seen in the controls, but GFPdnLMP1 expression could barely be detected in the transfected cultures at 3 weeks post transfection and was not detected by 4 weeks (not shown). Therefore earlier time points post transfection were examined. At two days post transfection of 3959.48 cells strong expression of GFPdnLMP1 was detected which was considerably reduced by 5 days post transfection and again only low level expression was detected by 3 weeks post transfection (despite plasmid selection), while control GFP expression in this cell line was constant (figure $5 \mathrm{C})$.

Thus, either GFPdnLMP1 expression (unlike GFP alone) becomes repressed in the 39.415 and 3959.48 transfected cells or those cells expressing the dominant negative LMP1 protein are lost from the culture. In order to examine the viability of the GFPdnLMP1 expressing cells in the transfected, selected cultures, 3959.48 cells at four weeks post transfection were stained with propidium iodide (PI; apoptotic cells stain with PI while viable cells exclude the stain) and examined by flow cytometry. Of the pGFPdnLMP1 transfected cells $0.8 \%$ showed GFP fluorescence, of which 76.3\% stained with PI (figure 5D). In contrast $6 \%$ of the pGFP transfected population showed GFP fluorescence of which $19.1 \%$ stained for PI. This suggests that the GFPdnLMP1 expressing cells were being eliminated from the population by apoptosis.

In order to look at earlier time points post transfection further, 39.415 and 3959.48 cells were examined by microscopy 24 hours after transfection. In these unselected cell populations bright fluorescent cells could clearly be seen in cultures transfected with both pGFP and pGFPdnLMP1, however there were fewer apparent in 


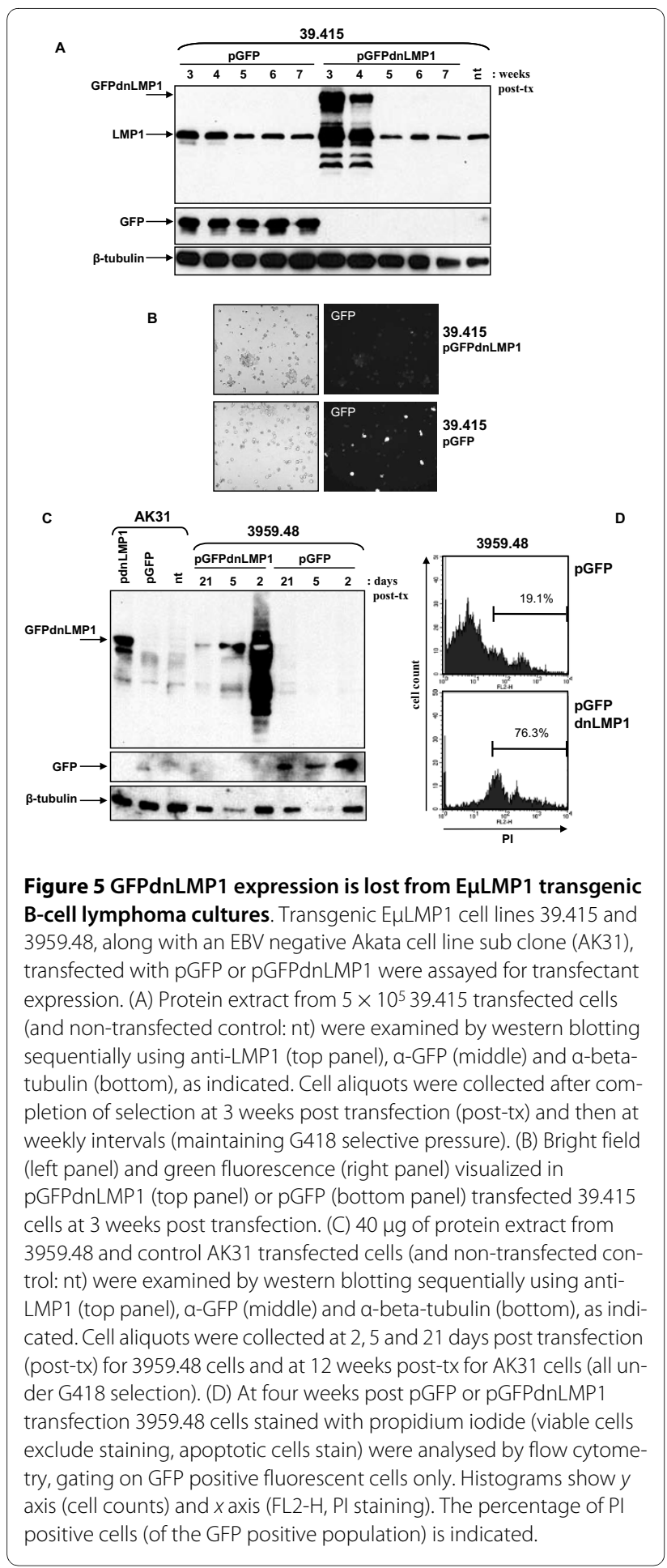

the latter and these often appeared morphologically unhealthy. In addition there was evidence of cells undergoing apoptosis in the pGFPdnLMP1 cultures (additional files $1 \& 2$, supplementary figure S6). GFP fluorescence in the transfected transgenic cells was also examined by flow cytometry. For cell line 39.415, the proportion of GFP expressing cells from 2 days post transfection to 5 days post transfection did not drop (36.9\% to $36.4 \%$ ). In contrast, the proportion of GFPdnLMP1 expressing cells dropped from $28.5 \%$ to $1.6 \%$ (figure 6). With 3959.48 cells 2 days post transfection, the proportion of GFP expressing cells was $6.6 \%$ compared to $2.1 \%$ for GFPdnLMP1 (figure 6).

These data demonstrate that both transgenic B-cell lines require the continued action of LMP1 for growth and survival, even in the cell line 3959.48 where LMP1 expression is very low.

\section{Discussion}

In this study we have examined the consequences of inhibiting LMP1 activity in several cell lines which were derived from transgenic mice where LMP1 was the driving oncogene in the tumourigenic process. A dominant negative mutant of LMP1 which inhibits its signalling capacity was used with a view to future therapeutic drugs which might target LMP1 function in a competitive manner. We have explored the effects of inhibition in cells from established tumours, not upon cancer development, to reflect that in the clinical setting treatment is only initiated in patients with established tumours. Furthermore, in a number of these cell lines, LMP1 expression was low or undetectable and its continued function in the tumour cells was equivocal.

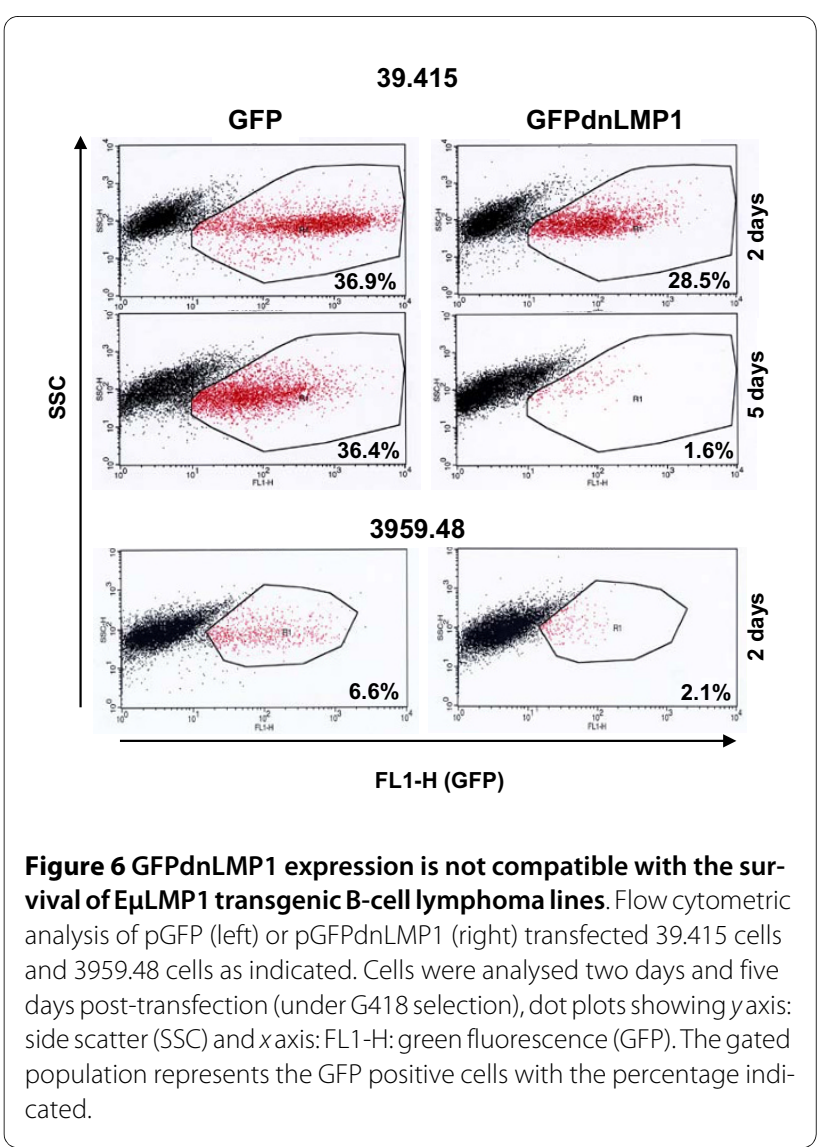


Inhibition of LMP1 activity in carcinoma cell lines established from LMP1 transgenic mice resulted in reduced clonagenicity (reflecting growth and survival of the cells at low density) in all of the cell lines tested. Interestingly, this was even the case in cell lines where LMP1 protein expression could not be detected, suggesting that there is a low-level (below detection) expression of LMP1 in these cell lines and that it still confers a growth advantage to the cells. This is relevant to NPC where LMP1 RNA can be detected in the majority of tumours but protein in only $30 \%$ to $50 \%$ of samples. This raises the possibility that the $50 \%$ to $70 \%$ of cases in which LMP1 protein is not detected may nevertheless express functioning LMP1.

Clonagenicity was not abolished in the carcinoma cell lines studied here (with the exception of one cell line). To varying degrees with the different lines, clones could be established and expression of dnLMP1 was shown for two LMP1 transgenic positive cell lines. Clone 53.234dnL-1 (derivative of 53.234a) must have undergone genetic or epigenetic changes to enable its establishment, but it nevertheless had slower growth characteristics than the parental cell line. As such, any changes it incurred did not fully compensate for LMP1 activity in the growth of the cell. By contrast 53.278adnL clones had more than compensated, evolving a faster growth pattern than the parental cell line. This cell line was derived from a spindle cell tumour, advanced and aggressive in nature and as such may have already undergone several changes in vivo to render LMP1 function redundant. One could therefore speculate that any LMP1 directed therapy with such a tumour (in the absence of other therapies) would force progression through selection, leading to drug resistant, aggressive escape mutants. Nevertheless, such a treatment could still potentially augment cytotoxic drug treatment.

With the cell lines and derived clones developed here we are now in a position to investigate the critical changes required in a tumour cell to allow it to overcome loss of LMP1 function. This knowledge could provide further targets to be used in combination with any LMP1 directed therapy.

The LMP1 transgenic B-cell lines examined could not tolerate inhibition of LMP1 activity, even in the 3959.48 cell line with very low LMP1 levels. Expression of dnLMP1 was either rapidly shut down in the transfected cells or those expressing cells were lost from the population. The kinetics would suggest the latter, as loss of dnLMP1 expression in the population could be seen after only a few population doublings, despite selection for plasmid retention. Moreover, after several weeks, the remaining GFPdnLMP1 positive cells showed evidence of undergoing apoptosis. With the loss of GFPdnLMP1 expression from the selected population, no expressing clones could be isolated. This is not to say that escape mutation is not possible in these B-cells, but perhaps occurs at too low a frequency to have been isolated in these assays.

Human EBV-associated tumours may have a more complex etiology than the transgenic tumours described here and indeed, several EBV-associated tumours show absolutely no expression of LMP1. However, in those EBV-associated tumours that do show LMP1 expression (if not consistently), like NPC and HD, it is reasonable to conclude from the wealth of data available on LMP1 activity, that it has been factorial in the development of the tumour, as it has in these transgenic mouse tumours. The data described here show that LMP1 continues to provide a growth advantage in established tumours even when expressed at very low level and provide proof of principle that in these tumour types LMP1 directed therapy could be effective.

\section{Conclusions}

Generally, therapeutic drug resistance emerges following cancer treatments as a function of the number of tumour cells at the time of treatment as well as their potential ability to overcome the treatment. As a viral protein LMP1 diverts cellular processes to affect an altered growth programme of the cell and by secretion of induced factors, alter the cellular environment. It is not a cellular product and therefore not fundamentally essential to the cell. There are likely to be multiple mutational routes (of cellular genes) which could compensate for the loss of LMP1 function in the tumour cell and thus multiple possible routes for resistant cells to emerge following any LMP1 directed therapy. However, our results suggest that inhibition of LMP1 could be highly effective with some tumours and possibly stall others, even in EBV associated cancer types where detection of LMP1 is inconsistent, such as NPC. If combined with cytotoxic drugs, targeting LMP1 action could improve outcome in both epithelial and B-cell tumours.

\section{Methods}

\section{E $\mu L M P-1$ and PyLMP1 transgenic lines and tumours}

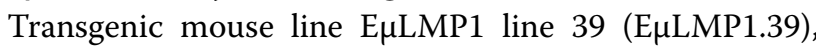
expressing low levels of LMP1 in the lymphoid compartment has been used in the studies described herein [3], maintained in the $\mathrm{C} 57 \mathrm{Bl} / 6$ strain. Mice of this line develop lymphoma at an average age of approximately 20 months (supplementary information figure 1). Lymphoma incidence in the transgenic mouse line E $\mu$ EBNA1.59 expressing EBNA-1 in the lymphoid compartment, has been previously described [17]. Mice of line PyLMP1.53 (LMP1 of the EBV B95-8) express LMP1 in the epidermis and are maintained in the FVB mouse strain [3,13]. Carcinomas were induced in PyLMP1.53 
mice using a standard single dose DMBA followed by 20 week TPA topical chemical carcinogen regime as previously described $[13,18]$.

\section{Cell lines}

Cell line 39.415 was developed following sequential in vivo passage of a B-cell tumour arising in mouse E $\mu L M P 1.39$ number 415. After 3 passages in B6D2 strain immunocompetent mice, the tumour could be established in culture (supplementary information). Cell line 3959.48, expressing both LMP1 and EBNA-1 was established in culture following explant of a B-cell lymphoma from a bitransgenic mouse of the lines E $\mu L M P 1.39$ and E $\mu$ EBNA-1.59. B-cell lines were grown in RPMI supplemented with $10 \%$ FCS, 2 mM glutamine, 100 units $/ \mathrm{ml}$ penicillin/streptomycin. Carcinoma cell lines were developed from primary carcinomas as described [13], grown in DMEM containing 10\% FCS, $2 \mathrm{mM}$ glutamine, 100 units/ml penicillin/streptomycin. CarB is a spindle-cell carcinoma cell line derived from a wild type mouse following DMBA/TPA chemical carcinogen treatment [19]. Raji is an EBV positive BL cell line, BL2B958 is an EBV negative $\mathrm{BL}$ cell line subsequently infected with $\mathrm{EBV}$ of the B95-8 strain, AK31 is an EBV negative derivative of the EBV positive Akata BL cell line.

\section{Protein extraction and western blotting}

Protease inhibitors (complete mini, Roche), $1 \mathrm{mM}$ phenylmethylsulfonyl fluoride and phosphatase inhibitors (10 $\mu \mathrm{l} / \mathrm{ml}$, phosphatase inhibitor cocktail II, Sigma) were freshly added to the protein extraction buffers. Proteins were extracted according to one of three protocols: [1] using urea protein extraction buffer (8M urea, $25 \mathrm{mM}$ tris- $\mathrm{HCl} \mathrm{pH9.5,} \mathrm{5 \%} \mathrm{(v/v)} \mathrm{2-mercaptoethanol),} \mathrm{with} \mathrm{incu-}$ bation at $55^{\circ} \mathrm{C}$ overnight with agitation; [2] using RIPA buffer $(150 \mathrm{mM} \mathrm{NaCl}, 50 \mathrm{mM}$ tris- $\mathrm{HCl} \mathrm{pH} 7.5,1 \%(\mathrm{v} / \mathrm{v})$ triton, $1 \%(\mathrm{w} / \mathrm{v})$ deoxycholic acid, $0.1 \%(\mathrm{w} / \mathrm{v})$ SDS) followed by sonication; [3] alternatively counted cells were resuspended in PBS with protease inhibitors and sonicated and an equal volume of $2 \times$ boiling mix was added $(1 \times$ boiling mix: $62.5 \mathrm{mM}$ tris- $\mathrm{HCl} \mathrm{pH} 6.8,2 \%(\mathrm{w} / \mathrm{v}) \mathrm{SDS}$, $5 \%(\mathrm{v} / \mathrm{v}) 2$-mercaptoethanol, $10 \%(\mathrm{v} / \mathrm{v})$ glycerol, trace bromophenol blue), heated to $95^{\circ} \mathrm{C}$ for 5 minutes for direct gel loading. Protein concentration was determined by Bradford assay (Biorad) or by 2D Quant assay (GE Healthcare). For SDS-PAGE, boiling mix was added to a $1 \times$ concentration to protein aliquots which were heated to $95^{\circ} \mathrm{C}$ for 5 minutes and loaded on to gels of $7.5 \%, 10 \%$ or $12.5 \%$. Gels were blotted and blots were probed and washed as previously described [18]. Blots were incubated in 5\% non-fat milk, $0.1 \%(\mathrm{v} / \mathrm{v})$ Tween 20 in PBS with either 1:1000 anti $\beta$-tubulin (sc-9104, Santa Cruz), 1:100 1G6 (anti-LMP1) [20] or 1:500 anti-GFP (sc-8334, Santa Cruz) followed by 1:4000 of the appropriate IgG-
HRP-conjugated secondary antibody (anti-rabbit sc2030, anti-rat sc-2032 Santa-Cruz) and visualized by enhanced chemiluminescence (liteAblot kit, Euroclone).

\section{Immunoprecipitation}

Equal quantities of urea-extracted protein samples were diluted at least 10-fold and made up to a total volume of 1 ml with NET-N pH8.0 (150 mM NaCl, 5 mM EDTA pH8, $50 \mathrm{mM}$ tris- $\mathrm{HCl} \mathrm{pH8}, 0.05 \%(\mathrm{v} / \mathrm{v}) \mathrm{NP}-40)$ including protease and phosphatase inhibitors. To pre-clear, $70 \mu \mathrm{l}$ of $50 \%(\mathrm{v} / \mathrm{v})$ protein sepharose G (pre-washed) in NET-N buffer was added to each of the samples and rotated at $4^{\circ} \mathrm{C}$ for 2 hours. The samples were centrifuged at $10000 \mathrm{~g}$ for 10 mins at $4^{\circ} \mathrm{C}$, and the pre-clear step was repeated with the supernatant using $30 \mu \mathrm{l}$ of $50 \%(\mathrm{v} / \mathrm{v})$ protein sepharose G. $4 \mu$ l of anti-LMP1 S12 [21] was added to the pre-cleared supernatant and rotated at $4{ }^{\circ} \mathrm{C}$ overnight. 30 $\mu \mathrm{l}$ of $50 \%(\mathrm{v} / \mathrm{v})$ protein sepharose $\mathrm{G}$ was added to each sample and rotated at $4^{\circ} \mathrm{C}$ for 30 mins. The samples were centrifuged at $10000 \mathrm{~g}$ for $10 \mathrm{mins}$ at $4^{\circ} \mathrm{C}$ and the pellet was washed with $1 \mathrm{ml}$ of NET-N pH8.0, followed by $1 \mathrm{ml}$ of PBS with centrifugation at $10000 \mathrm{~g}$ for $1 \mathrm{~min}$ at $4^{\circ} \mathrm{C}$. The antibody-antigen complexes were eluted from the beads with $30 \mu \mathrm{l}$ of boiling mix at $95^{\circ} \mathrm{C}$ for $5 \mathrm{mins}$ and centrifuged at $10000 \mathrm{~g}$ for $1 \mathrm{~min}$ prior to SDS-PAGE.

\section{Plasmids and transfection}

The dominant-negative LMP1 plasmid pGFPdnLMP1 encoding an LMP1AAAG mutant in which codons 204, 206, 208 and 384 have been changed from amino acids $P$, $\mathrm{Q}, \mathrm{T}$ and $\mathrm{Y}$ to $\mathrm{A}, \mathrm{A}, \mathrm{A}$ and $\mathrm{G}$ and linked at the $\mathrm{N}$-terminus to an in-frame enhanced-GFP tag, under the control of the CMV promoter, has been previously described [16]. It is abbreviated to dnL for cell subclones transfected with the plasmid. As control, pEGFP-C1 (Clontech) (abbreviated here to pGFP) encoding enhanced-GFP under the control of the CMV promoter has been used. B cells (typically $1 \times 10^{7}$ ) were transfected with $10 \mu \mathrm{g}$ of plasmid DNA by electroporation (960uF, $250 \mathrm{~V}$ ), or "no DNA" as control, using a Biorad electroporater or an Amaxa nucleofector with solution V. One day after transfection cells were subjected to G418 selection (supplementary information table 1) and regarded as stably transfected (or post the selective process) when all no DNA controls cells were dead ( 2 to 3 weeks post transfection). Post selection cells were continually maintained in G418 thereafter. Epithelial cell lines were transfected in duplicate with either superfect (Qiagen) or metafectene lipid-based transfection reagents according to the manufacturer's instructions. Typically, one day after transfection cells were split $1: 8$ and then subjected to selection which was usually complete by 2 weeks (supplementary information table 1). Post selection clones were continually maintained in G418 thereafter. 


\section{Clonagenicity assay with crystal violet}

Cells were plated in $6 \mathrm{~cm}$ dishes, transfected with the appropriate plasmid and selected with G418. 14 days post-transfection, surviving colonies were stained with crystal violet solution $(0.5 \%(\mathrm{w} / \mathrm{v})$ crystal violet, $20 \%(\mathrm{v} / \mathrm{v})$ ethanol in $\mathrm{dH}_{2} \mathrm{O}$ ) at RT for 10 mins to 1 hour, washed gently with tap water and allowed to dry. The number of clones on each plate was counted directly.

\section{Cell growth assay with neutral red}

Cells were seeded at a density of 500 cells per well (in quadruplicate sets for each daily count) in 96-well plates in $100 \mu \mathrm{l}$ of medium. At daily intervals, cells were treated as follows: the medium was replaced in the wells to be analysed with pre-warmed neutral red-containing $(40 \mu \mathrm{g} /$ $\mathrm{ml}$ ) medium and incubated at $37^{\circ} \mathrm{C}, 5 \% \mathrm{CO}_{2}$ for 3 hours. The medium was removed, the cells were fixed with 100 $\mu \mathrm{l}$ of $1 \%(\mathrm{w} / \mathrm{v}) \mathrm{CaCl}_{2}, 0.5 \%(\mathrm{v} / \mathrm{v})$ formaldehyde which was then removed and $100 \mu \mathrm{l}$ of $1 \%(\mathrm{v} / \mathrm{v})$ acetic acid $50 \%(\mathrm{v} / \mathrm{v})$ ethanol was added to each well in order to liberate the dye from the viable cells that had incorporated stain. The plate was incubated at RT for 10-15 mins, rocked for 2030 mins, then neutral red containing solutions were transferred to an empty plate and the absorbance was measured at $540 \mathrm{~nm}$. Cells were assayed up to confluence (6 to 8 days). Statistical difference was calculated using a two sample T-test assuming equal variances.

\section{Flow cytometry}

Cells were analysed using a FACScalibur flow cytometer (Becton Dickinson). Data was collected and analysed using CellQuest software (Becton Dickinson). Where possible, 10000 events (or GFP positive cells) were analysed. For GFP analysis cells were counted, washed twice with PBS and resuspended at $2 \times 10^{6}$ cells $/ \mathrm{ml}$ in PBS prior to FACS analysis. Apoptosis was assessed by propidium iodide exclusion; cells were counted, washed twice with PBS and resuspended at $2 \times 10^{6} \mathrm{cells} / \mathrm{ml}$ in PBS. Typically $2 \times 10^{6}$ to $1 \times 10^{7}$ cells were used. Immediately prior to FACS analysis, $10 \mu \mathrm{l}$ of $50 \mathrm{mg} / \mathrm{ml}$ propidium iodide (PI) solution was added per $1 \mathrm{ml}$ of cell suspension.

\section{Additional material}

\section{Additional file 1 This file contains the legends to the supplementary} figures (S1 to S6) and supplementary table S1.

Additional file 2 This file contains supplementary figures $S 1$ to $S 6$ and supplementary table $\mathrm{S} 1$.

\section{Competing interests}

The authors declare that they have no competing interests.

\section{Authors' contributions}

AH carried out the studies described, contributed to their design and helped to draft the manuscript. JBW conceived, coordinated and designed the study, performed the statistical analysis and drafted the manuscript. Both authors read and approved the final manuscript.

\section{Acknowledgements}

Thanks go to Martin Rowe for the kind gift of plasmid pEGFP/LMP1AAAG referred to here as pGFPdnLMP1. We thank Asif Qureshi who conducted the western shown in figure 1B. We thank Tom Gilbey for assistance with flow cytometry. We thank Nooshin Dabbagh, Mark Drotar and Helen Bryden who developed the two B-cell lines 39.415 and 3959.48 used here. This work was supported by the Wellcome Trust (\#069113/Z/02).

\section{Author Details}

'Division of Molecular and Cellular Biology, Faculty of Biomedical and Life Sciences, University of Glasgow, Glasgow G11 6NU, UK and ${ }^{2}$ Current address: EnGenelC Ltd, Sydney, NSW 2066, Australia

Received: 9 March 2010 Accepted: 9 July 2010

Published: 9 July 2010

\section{References}

1. Crawford DH: Biology and disease associations of Epstein-Barr virus. Philos Trans R Soc Lond B Biol Sci 2001, 356:461-473.

2. Kieff E, Rickinson AB: in Fields Virology. Edited by: Knipe DM, Howley PM, Griffin DE. Lippincott Williams \& Wilkins, Philadelphia; 2001:2511-2573.

3. Wilson JB, Weinberg W, Johnson R, Yuspa S, Levine AJ: Expression of the BNLF-1 oncogene of Epstein-Barr virus in the skin of transgenic mice induces hyperplasia and aberrant expression of keratin 6. Cell 1990, 61:1315-1327.

4. Kulwichit W, Edwards RH, Davenport EM, Baskar JF, Godfrey V, Raab-Traub $\mathrm{N}$ : Expression of the Epstein-Barr virus latent membrane protein 1 induces B cell lymphoma in transgenic mice. Proc Natl Acad Sci USA 1998, 95:11963-11968.

5. Stevenson D, Charalambous C, Wilson JB: Epstein-Barr Virus Latent Membrane Protein 1 (CAO) Up-regulates VEGF and TGFa Concomitant with Hyperlasia, with Subsequent Up-regulation of p16 and MMP9. Cancer Res 2005, 65:8826-8835.

6. Young LS, Murray PG: Epstein-Barr virus and oncogenesis: from latent genes to tumours. Oncogene 2003, 22:5108-5121.

7. Miller WE, Earp HS, Raab-Traub N: The Epstein-Barr virus latent membrane protein 1 induces expression of the epidermal growth factor receptor. J Virol 1995, 69:4390-4398.

8. Charalambous CT, Hannigan A, Tsimbouri P, McPhee GM, Wilson JB: Latent membrane protein 1 -induced EGFR signalling is negatively regulated by TGF alpha prior to neoplasia. Carcinogenesis 2007, 28:1839-1848.

9. Tsai J, Lee JT, Wang W, Zhang J, Cho H, Mamo S, Bremer R, Gillette S, Kong J, Haass NK, et al: Discovery of a selective inhibitor of oncogenic B-Raf kinase with potent antimelanoma activity. Proc Natl Acad Sci USA 2008, 105:3041-3046.

10. Young LS, Rickinson AB: Epstein-Barr virus: 40 years on. Nat Rev Cancer 2004, 4:757-768

11. Lo AK, To KF, Lo KW, Lung RW, Hui JW, Liao G, Hayward SD: Modulation of LMP1 protein expression by EBV-encoded microRNAs. Proc Natl Acad SciUSA 2007, 104:16164-16169.

12. Mei YP, Zhou JM, Wang Y, Huang H, Deng R, Feng GK, Zeng YX, Zhu XF: Silencing of LMP1 induces cell cycle arrest and enhances chemosensitivity through inhibition of AKT signaling pathway in EBVpositive nasopharyngeal carcinoma cells. Cell Cycle 2007, 6:1379-1385.

13. Curran JA, Laverty FS, Campbell D, Macdiarmid J, Wilson JB: Epstein-Barr virus encoded latent membrane protein-1 induces epithelial cell proliferation and sensitizes transgenic mice to chemical carcinogenesis. Cancer Res 2001, 61:6730-6738.

14. Tsimbouri P, Al-Sheikh Y, Drotar ME, Cushley W, Wilson JB: Epstein-Barr virus nuclear antigen-1 renders lymphocytes responsive to IL-2 but not IL-15 for survival. J Gen Virol 2008, 89:2821-2832.

15. Salcedo R, Fuerstenberg SM, Patarroyo M, Winberg G: The Epstein-Barr virus BNLF-1 membrane protein LMP1 induces homotypic adhesion mediated by $\mathrm{CD} 11 \mathrm{a} / \mathrm{CD} 18$ in a murine B-cell line, mimicking the action of phorbol ester. J Virol 1991, 65:5558-5563.

16. Brennan P, Floettmann JE, Mehl A, Jones M, Rowe M: Mechanism of action of a novel latent membrane protein-1 dominant negative. J Biol Chem 2001, 276:1195-1203.

17. Wilson JB, Bell JL, Levine AJ: Expression of Epstein-Barr virus nuclear antigen-1 induces B cell neoplasia in transgenic mice. EMBO J 1996, $15: 3117-3126$ 
18. Macdiarmid J, Stevenson D, Campbell DH, Wilson JB: The latent membrane protein 1 of Epstein-Barr virus and loss of the INK4a locus: paradoxes resolve to cooperation in carcinogenesis in vivo. Carcinogenesis 2003, 24:1209-1218.

19. Diaz-Guerra M, Haddow S, Bauluz C, Jorcano JL, Cano A, Balmain A, Quintanilla M: Expression of simple epithelial cytokeratins in mouse epidermal keratinocytes harboring Harvey ras gene alterations. Cancer Res 1992, 52:680-687.

20. Nicholls J, Hahn P, Kremmer E, Frohlich T, Arnold GJ, Sham J, Kwong D, Grasser FA: Detection of wild type and deleted latent membrane protein 1 (LMP1) of Epstein-Barr virus in clinical biopsy material. J Virol Methods 2004, 116:79-88.

21. Mann KP, Staunton D, Thorley-Lawson DA: Epstein-Barr virus-encoded protein found in plasma membranes of transformed cells. J Virol 1985, 55:710-720.

doi: 10.1186/1476-4598-9-184

Cite this article as: Hannigan and Wilson, Evaluation of LMP1 of Epstein-Barr virus as a therapeutic target by its inhibition Molecular Cancer 2010, 9:184

Submit your next manuscript to BioMed Central and take full advantage of:

- Convenient online submission

- Thorough peer review

- No space constraints or color figure charges

- Immediate publication on acceptance

- Inclusion in PubMed, CAS, Scopus and Google Scholar

- Research which is freely available for redistribution

Submit your manuscript at www.biomedcentral.com/submit
C) Biomed Central 